\title{
A Simple Effective Filtering Method for Removing CPR Caused Artefacts from Surface ECG Signals
}

\author{
E Aramendi, J Ruiz, S Ruiz de Gauna, U Irusta, A Lazkano, J Gutierrez \\ University of the Basque Country, Bilbao, Spain
}

\begin{abstract}
Cardiopulmonary Resuscitation (CPR) introduces strong interference on the surface ECG, corrupting any diagnosis of the cardiac rhythm.

In this work a filtering method is proposed to remove the CPR artefact from Ventricular Fibrillation (VF) signals. It is a notch filter adapted to the fundamental frequency of the CPR artefact. It is designed using the mean time interval between compressions characteristic of each CPR artefact.

The method has been tested with a set of 17 artefact signals and 191 VF signals, recorded in real out-ofhospital assistances. For the different corruption levels tested (i.e. SNR of -10, -6, -3, 0, 3 and $3 \mathrm{~dB}$ ), the mean SNR values restored are: $-3.16,0.39,2.77,4.79$ and 6.38 $d B$. The positive effect of the filtering on the rhythm classification algorithm of an AED has been evaluated. The sensitivities values after filtering are: $52.26 \%$, $79.3 \%, 90.48 \%, 94.91 \%$ and $96.64 \%$ respectively.
\end{abstract}

\section{Introduction}

The actions described as essential in the Chain of Survival for the intervention of Emergency Medical Services are: early access, early cardiopulmonary resuscitation, early defibrillation and early advance care [1]. The CPR is the best treatment for cardiac arrest until the arrival of an AED as it prevents VF from deteriorating to asystole. Moreover, longer compression periods during CPR may increase the rate of restoration of spontaneous circulation (ROSC). But the mechanical chest compressions that CPR implies, introduce severe artefacts in the surface ECG signal. These artefacts severely condition the shock/no-shock decision of an AED, so CPR has to be interrupted during the automatic rhythm analysis. Evidently the duration of these 'hands-off' intervals affects negatively to the rate of ROSC. This work deals with the challenge of removing the CPR artefact and detecting reliably the VF signal.

The CPR artefacts have been successfully removed from porcine ECG by means of fixed coefficient filters [2]. Due to the important spectral overlap that human VF and CPR show, and due to the high variability of the CPR artefact a constant filter is not an adequate approach for the human case [2-4]. Recent studies propose adaptive filters that require several additional signals strongly correlated to the artefact, i.e. thoracic impedance, compression acceleration, compression depth, ... [5]. These signals are not available in current AEDs and they require strong hardware development to be obtained.

In this study we propose a filtering technique that is based on the knowledge of the mean interval between thoracic compressions during the CPR. The notch filter is designed adapted to the precise fundamental frequency associated to each CPR artefact.

The filtering technique is tested with a set of 17 artefact signals caused by CPR on patients in asystole, combined with a set of $191 \mathrm{VF}$ samples with different corruption levels $(\mathrm{SNR}=-10,-6,-3,0$ and $6 \mathrm{~dB})$. The performance of the method is evaluated on one hand computing the SNR of the VF signal after removing the artefact with the notch filter. On the other hand, the sensitivity of the 'shock/no-shock' algorithm of an AED is computed for the test database before and after filtering.

\section{Methods}

The set of CPR artefact consist of 17 samples that were recorded in real out-of-hospital assistances. Each sample corresponds to the surface ECG recorded on a different patient in asystolic rhythm when thoracic compressing was being performed by different ambulance assistants.

The VF database used in this work was collected from different sources (hospital and emergency services) and fulfils the AHA requirements for the evaluation of the arrhythmia analysis algorithm of an AED [6]. A total of 191 segments of coarse VF were obtained with an average duration of 15 seconds. A detailed description of this database is provided in [7].

Following a widely applied model, each CPR artefact is added to every ECG signal with a given SNR. In Fig. 1 the original VF and the one corrupted by the CPR artefact are shown considering a SNR of $-3 \mathrm{~dB}$. In the second plot corresponding to the CPR artefact, the instants of every 
compression and the pause interval are evident. Dotted lines show the instants of compression that our method detects.

The time intervals between compressions and the pause duration are highly variable in the set of CPR artefact samples. Therefore the proposed method accounts for the CPR frequency variability through the value of the mean time interval between compressions.

The instant of every chest compression is set in the artefact signal detecting significant zero crossings. Intervals with no compression activity are detected based on duration, and they are not considered to compute the mean time interval between compressions. That way the number of compressions per minute is computed and the fundamental frequency value, $\mathrm{f}_{\mathrm{o}}$, corresponding to that artefact signal is estimated. In the example of Fig. 1 a value of $\mathrm{f}_{\mathrm{o}}=2.23 \mathrm{~Hz}$, i.e. a sequence of 133.8 compressions per minute is computed. The fundamental frequency values computed for the set of $17 \mathrm{CPR}$ samples analyzed vary from $1.7 \mathrm{~Hz}$ to $2.8 \mathrm{~Hz}$.
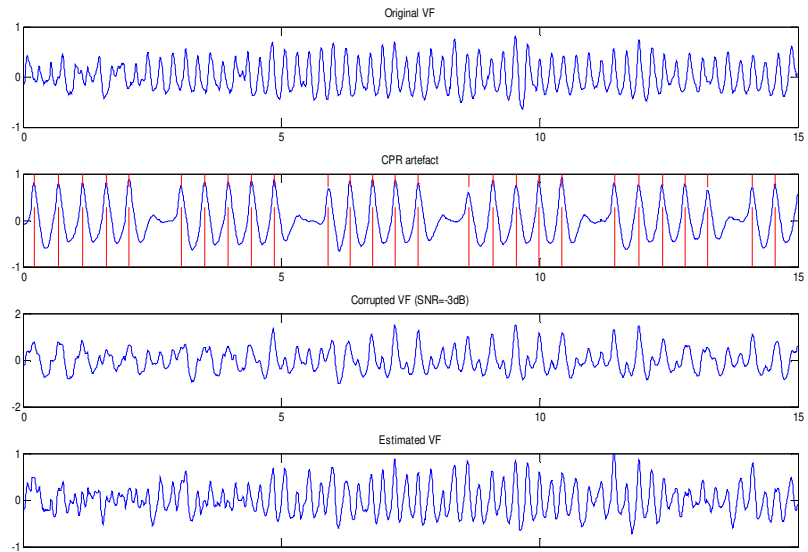

Figure 1. An example of adaptive notch filtering for CPR removing

The filtering technique proposed is a single notch filter adapted to each artefact. It is a second order notch filter centered on the $f_{o}$ of each artefact sample. It is designed using the fundamental frequency, $\mathrm{f}_{\mathrm{o}}$, and the $3 \mathrm{~dB}$ bandwidth $\mathrm{B}_{\mathrm{f}}$ following this characteristic:

$$
\mathrm{H}(\mathrm{z})=\frac{1-2 \cos \Omega_{0} \mathrm{z}^{-1}+\mathrm{z}^{-2}}{1-2\left(1-\mathrm{B}_{\omega} / 2\right) \cos \Omega_{0} \mathrm{z}^{-1}+\left(1-\mathrm{B}_{\omega} / 2\right)^{2} \mathrm{z}^{-2}}
$$

being,

$$
\begin{aligned}
& \Omega_{0}=2 \Pi \mathrm{f}_{\mathrm{o}} / \mathrm{f}_{\mathrm{s}} \\
& \mathrm{B}_{\omega}=2 \Pi \mathrm{B}_{\mathrm{f}} / \mathrm{f}_{\mathrm{s}}, \text { with Bf=1.2 Hz (optimun for the set) } \\
& \mathrm{f}_{\mathrm{s}}=250 \mathrm{~Hz}
\end{aligned}
$$

Fig. 2 shows the spectral characteristic of the notch filter for the CPR artefact analyzed in Fig. 1, (centered in $2.23 \mathrm{~Hz}$ ). The non-constant phase delay information, also shown in Fig. 2, is used to adjust the delay introduced by the filter in the $3.5-6 \mathrm{~Hz}$ band.
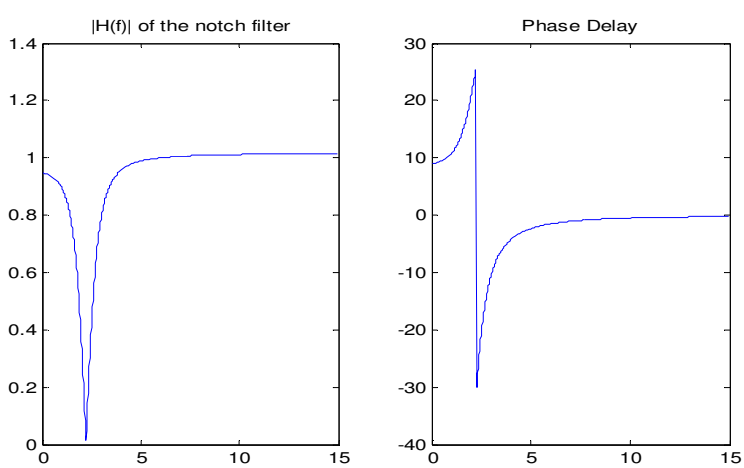

Figure 2. Frequency characteristic of the notch filter.

\section{Results}

To evaluate the proposed method, each of the 17 samples of CPR has been combined with each of the 191 VF samples with different SNR level (-10, -6, -3, 0 and 3 $\mathrm{dB})$. A total of 17 sets of corrupted VF have been obtained and the mean SNR restored is computed. Fig. 3 shows the performance of the filtering method for the $\mathrm{CPR}$ no. indicated in $\mathrm{x}$ axis. Although the performance depends on the artefact no. considered, a mean increase of $6.84,6.38,5.77,4.79$ and $3.38 \mathrm{~dB}$ is obtained respectively per each SNR tested.

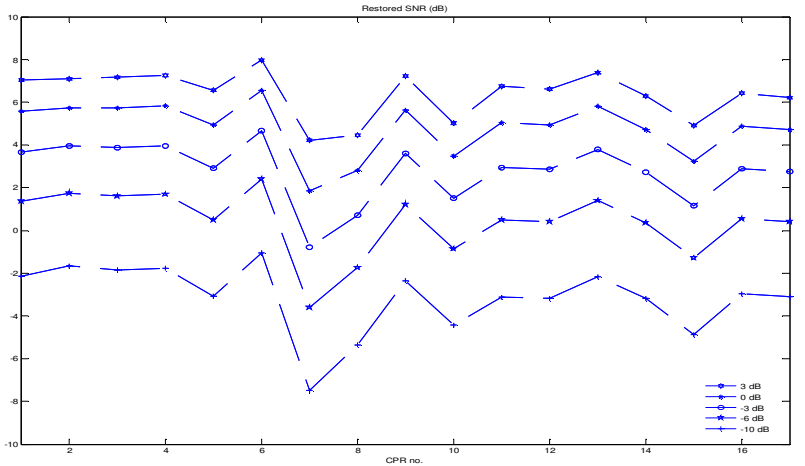

Figure 3. SNR restored for each of the artefact samples (1 to 17$)$.

These results have been compared with those obtained with the same filtering technique but using a different tuning frequency for the filter. Instead of using the $f_{o}$ reference value obtained from the CPR artefact, it has been considered the main frequency of the test signal in the 1-3 Hz band as it was proposed in [8]. As expected the performance using $f_{o}$, extracted from the instants of compression, are better in 2.07, 2, 1.89, 1.73 and $1.53 \mathrm{~dB}$ respectively for the tested SNR levels. 
a)

A more strict validation of the artefact removal technique is developed considering the response of the shock advice algorithm of an AED to the database of filtered VF samples. The original VF is a shockable rhythm which may be wrongly classified by the AED algorithm when corrupted by the CPR artefact. The filtering technique should restore this reliability providing an estimated VF that is classified as shockable. The sensitivity of the AED algorithm applied to the VF database before and after filtering will be used to measure the goodness of the proposed technique.

The AED shock advice algorithm used is an offline Matlab version of the one implemented in the Reanibex 200 desfibrillator (Osatu S.Coop., Spain) which uses two to three 4.8 second signal segment for a final 'shock/nonshock' decision. The results of sensitivity obtained for the five different SNR levels are shown in the plots of Fig. 4.

\section{Discussion and conclusions}

In this work a quite simple filtering technique has been analyzed and tested for removing the artefacts caused by CPR from ECG signals. It is a single notch filter which adapts its frequency characteristics to each CPR artefact, as it is tunned to the mean frequency of thoracic compressions $\left(f_{0}\right)$. This value is extracted from the CPR artefact as a reference value.

CPR artefacts and VF databases from humans have been used to evaluate the methods and restored SNR values have been computed. Although the mean increase of SNR is satisfactory, it is highly dependent on the CPR artefact considered. The mean increase values computed are $6.84,6.39,5.77,4.79$ and $3.38 \mathrm{~dB}$. These results have been compared to the same filtering not considering the reference value $f_{0}$. Results evidence that using the value extracted from the instants of chest compression instead of estimating it exclusively from the corrupted VF signal the method has a better performance.

As it can be observed in Fig. 3 the performance of the method decays for the CPR artefacts numbered as 7 and 8. Remembering the spectral analysis performed in [4] those two samples present high harmonic content, mainly in the second and the third harmonic. So, the next approach we are considering is a multiband notch filter that considers not only the content in $f_{o}$, but also the second and even the third harmonic.

Observing the sensitivity results shown in Fig. 4, it is evident the improvement that filtering provokes. The sensitivity after filtering is higher in all cases except in a couple of artefacts for $\mathrm{SNR}=-10 \mathrm{~dB}$, where the filter has removed frequency components which are essential for the 'shock/non-shock' decision algorithm.

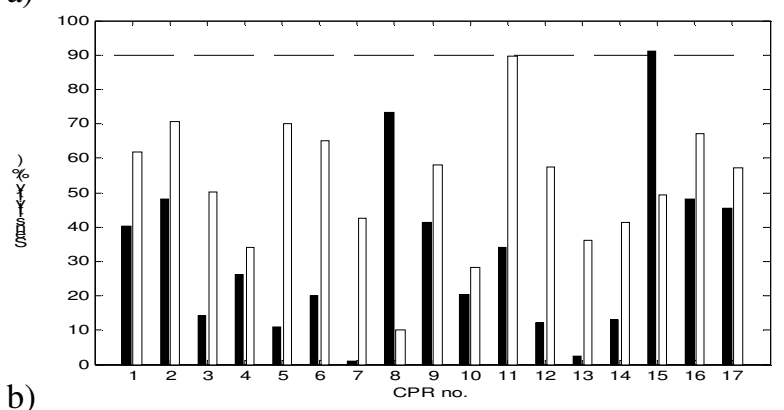

b)

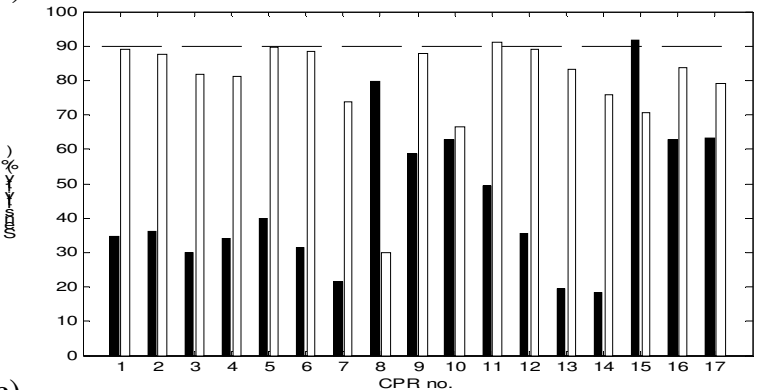

c)

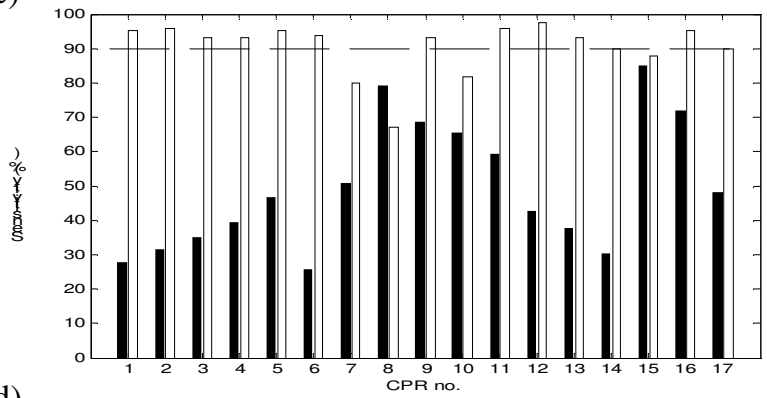

d)

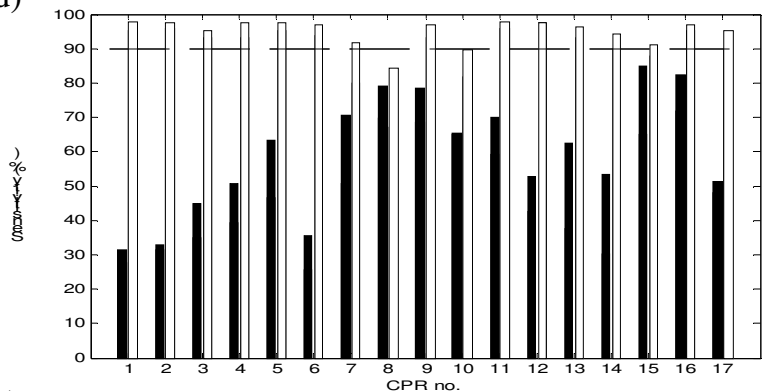

e)

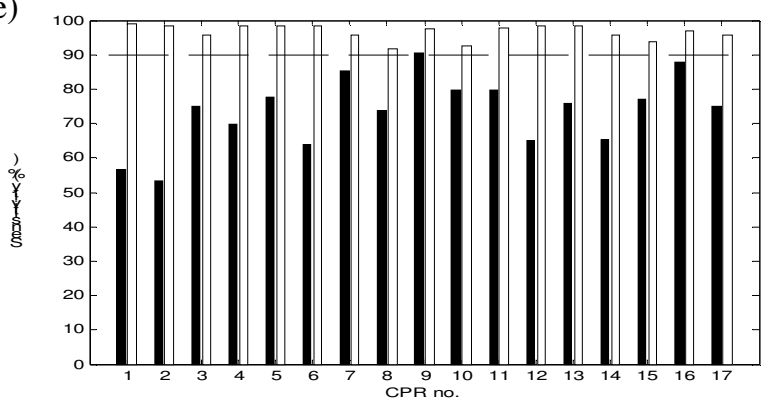

Figure 4. Sensitivity results for $-10 a$ a), -6 b), -3 c), 0 d) and $3 \mathrm{~d}) \mathrm{dB}$, before (black) and after (white) filtering. 
Summarily, the sensitivity improvement assures a value higher than $90 \%$ practically in all cases for the corruption levels of $-6,-3,0$ and $3 \mathrm{~dB}$. That makes the estimated VF database fulfil the requirement of AHA that sets in $90 \%$ the percentage of course VF that should be correctly classified as shockable. This is a promising result in the challenging purpose of not interrupting AED analysis during CPR in emergency assistance.

\section{Acknowledgements}

The authors would like to thank the support of the MCYT of Spain through the project TIC2003-08460.

\section{References}

[1] American Heart Association in collaboration with International Liaison Committee Resuscitation. Guidelines 2000 for Cardiopulmonary Resuscitation and Emergency Cardiovascular Care: International Consensus on Science, Part4: The Automated External Defibrillator: Key Link in the Chain of Survival. Circulation 2000; 102: I-60:I-72.

[2] Strohmenger HU, Lindner KH, Keller A, Lindner IM, Pfenninger EG. Spectral analysis of ventricular fibrillation and closed-chest cardiopulmonary resuscitation. Resuscitation 1996; 33(2): 155-61.

[3] Langhelle A, Eftest $\varnothing 1$ T, Myklebust H, Eriksen M, Holten BT, Steen PA. Reducing CPR artefacts in ventricular fibrillation in vitro. Resuscitation 2001. 48(3): 279-91.

[4] Ruiz de Gauna S, Aramendi E, Ruiz J, Gutierrez JJ. Characterization of cardiopulmonary resuscitation artefact on human ventricular fibrillation. Proceedings of the 17-th Biennial Intermational Eurasip Conference BIOSIGNAL 2004; 56-9.

[5] Eilevstjønn J, Eftestøl T, Aase SO, Myklebust H, Husøy JH, Steen PA. Feasibility of shock advice analysis during CPR through removal of CPR artefacts from the human ECG. Resuscitation 2004; 61(2): 131-41.

[6] AHA Science Advisory and Coordination Committee. Automatic External Defibrillators for public access defibrillation: Recommendations for specifying and reporting arrhythmia analysis algorithm performance, incorporating new waveform, and enhancing safety. Circulation 1997, vol 95, p. 1677-1682.

[7] Aramendi E, Ruiz J, Lazkano A, Leturiondo L, Varona S. Construcción de una base de señales ECG para el análisis y validación del algoritmo de detección de ritmos desfibrilables de un DEA. Proceedings of the Conference URSI 2002;3-4.

[8] Aramendi E, Ruiz J, Ruiz de Gauna S, García B, Gutierrez JJ. Adaptive filtering applied to CPR artefact supresión in corrupted VF signals. Proceedings of the 17-th Biennial International Eurasip Conference BIOSIGNAL 2004; 84-7.
Address for correspondence:

Elisabete Aramendi Ecenarro

Escuela Superior de Ingeniería. Alda Urquijo s/n 48013, Bilbao, Spain

jtparece@bi.ehu.es 